\title{
Development and Performance Investigation of a Unique Dual-rotor Savonius-type Counter-rotating Wind Turbine
}

\author{
Djamal Hissein Didane ${ }^{1 *}$, Do'arsherah Kudam², Mohd Fadhli Zulkafli ${ }^{2}$, Sofian \\ Mohd $^{2}$, Mohd Faizal Mohideen Batcha ${ }^{1}$, Amir Khalid',
}

${ }^{1}$ Center for Energy and Industrial Environment Studies (CEIES),

Universiti Tun Hussein Onn Malaysia 86400 Parit Raja, Batu Pahat, Johor, Malaysia

${ }^{2}$ Department of Aeronautical Engineering,

Universiti Tun Hussein Onn Malaysia, 86400 Parit Raja, Batu Pahat, Johor, Malaysia

*Corresponding Author

DOI: https://doi.org/10.30880/ijie.2021.13.06.008

Received 22 May 2020; Accepted 31 January 2021; Available online 31 August 2021

\begin{abstract}
Wind power is sustainable and prevalent virtually all over the globe. However, the conversion efficiency of the conventional single-rotor wind turbine (SRWT) is still far from satisfactory. The dual-rotor counter-rotating concept is among the reliable techniques used to enhance the efficiency of a wind energy conversion device for its renowned effectiveness. This study aims to investigate the performance of a Savonius dual/twin-rotor system, particularly in low-speed wind conditions while employing the counter-rotating technique. The evaluation of this technique is presented in terms of aerodynamic characteristics, including the power and torque coefficients. The results have shown that the new concept was able to improve the performance of the system extensively and was capable of operating in a lower wind speed condition. Compared to a single-rotor system, an additional $42 \%$ more torque was possible owing to the existence of a second rotor in the new system. The results have also revealed that the conversion efficiency of the system has been enhanced substantially. A corresponding average power coefficient of up to $28 \%$ was achieved. The present technique is thought to be promising for wind energy conversion systems, including sites with poor wind conditions.
\end{abstract}

Keywords: Counter-rotating technique, savonius rotor, Vertical axis wind turbine (VAWT), wind turbine design, low-speed wind

\section{Introduction}

Renewable energy sources are abundant, yet, the conversion efficiency of the existing devices is still regarded as relatively low. Wind power, in particular, is still not exploited in a satisfactory manner. This could mainly be owed to the fact that although according to Betz theory, the efficiency of a wind turbine can go up to 59.3\%, currently, the most efficient conventional single-rotor wind turbine (SRWT) system can extract about one-third only, regardless of the direction of the rotational axis, i.e. horizontal axis or a vertical axis. Such inability is viewed from two different standpoints. One is related to geographical conditions such as deciding a proper site for installation and the other is related to technical design issues such as design and fabrication of an efficient rotor and generator [1]. Although both matters may play a significant role in the amount of harnessed energy, the first issue, however, could be regarded as more mature compared to the latter one. Hence, considerable efforts have been exerted today to improve the efficiency of the two prominent designs of wind turbine i.e. horizontal axis wind turbine (HAWT) and vertical axis wind turbine (VAWT), using numerical simulations as well as active and passive experimental methods. These efforts include 
improving the design of the airfoil [2]-[8], the generator performance [9][10], or introducing a second additional rotor alongside the existing rotor in the system for better performance [11]-[17]. In the latter case, the new rotor is meant to spin in the opposite direction of the existing rotor for optimum operation. Such a technique is known as the counterrotating concept. A number of studies have confirmed the effectiveness of the counter-rotating technique, including [11]-[17] for applications in both HAWT and VAWT. A maximum conversion efficiency of up to 40\% [11] in terms of power was attainable for wind turbines with a vertical axis and an additional more than 60\% [17] power was possible for wind turbines with a horizontal axis, which provides strong evidence for the efficacy of this method for both types of wind turbine designs.

However, although prior studies have noted the importance of the counter-rotating wind turbine (CRWT) concept in improving the conversion efficiency of a wind turbine, the other major challenge encountered while harvesting wind energy is operating in a low-speed wind condition. Typically, the starting speed for most of the existing designs of wind turbines is considered relatively high for numerous sites which naturally require a higher rating speed for the wind turbine. This may not be an issue for terrains with strong wind, however, it is a real concern for sites in the equatorial zones in particular, due to the low annual average wind speed in this region which is in the range of $2 \mathrm{~m} / \mathrm{s}$ to $5 \mathrm{~m} / \mathrm{s}$ [18], [19]. As a result, the application of most wind turbines is inconveniently limited to such sites in order to be feasible commercially. As such, further work is required to establish the viability of the counter-rotating technique in a lower operating wind condition. The current design is developed in an effort to improve the performance of the innovative concept of the counter-rotating VAWT which was presented by Didane et al., [11] but this time using a Savonius-type rotor.

The Savonius-type rotor is renowned for its inherent capabilities of operating at a lower wind speed compared to other types of VAWT rotors. It is also characterized by its high torque and low efficiency. This low efficiency is far below the Betz limit (59.3\%); less than a half, which resulted in less attractiveness and competitiveness compared to other types of VAWT in terms of aerodynamics performance [20], [21]. Nevertheless, it is still being considered and adopted, particularly in developing countries in do-it-yourself projects for its easy fabrication. However, numerous studies claim that the efficiency of a Savonius turbine rotor could be enhanced and can reach up to $30 \%$ at a low rotational speed. It was, however, proved in an extensive study performed by Sandia [22] that at higher rotational speed, the efficiency of such rotor decreases dramatically. Thus, such a turbine is only suitable for low-speed conditions. Therefore, this design is usually found in high torque and low-speed applications such as water pumping and ventilation.

The counter-rotating concept, as shown in Figure 1, is a technique that requires the rotation of two rotors in completely opposite directions. Although it is a widespread technique in HAWT, nevertheless, it has just started to take a turn in VAWT applications. Unlike HAWT, the main advantage of this concept on VAWTs is that both rotors receive a similar up-stream wind speed and not only the escaped wind speed from the front rotor goes to the rear rotor as is the case in a horizontal axis CRWT. As a result, a comparable output is usually expected from both rotors due to the exposure to a similar strength wind speed. Therefore, the current counter-rotating concept is thought to be more advantageous in VAWT applications than in HAWT in terms of improving the overall output of a single-rotor system.

While it could be perceived that in terms of operation, the current counter-rotation in VAWT would be similar to the counter-rotation in HAWT, however, it is emphasized that this not the case. It is also not just a series connection of rotors like conventional power generation. The operation of the present counter-rotating design requires shell running (rotation of the generator itself) along with the bottom rotor and the top rotor spins the shaft in opposite direction. This is because it is not possible to have one rotor at the top spinning the shaft and another rotor at the bottom with another shaft. This will require a second generator and a shaft. Given that the main advantage of this concept is to eliminate the need to operate with two generators and/or two shafts, there is no point in using them with this concept though it is the case in horizontal axis CRWT. Moreover, having two generators and two shafts would also create complications during installation since the counter-rotating rotors would eventually need a support such as a tower to sit upon while spinning. As such, the current technique does not use two generators nor two shafts, though two rotors were involved. The bottom rotor is literally attached to the generator which technically spins the magnetic coil alongside the rotor and the top rotor is attached to the shaft and therefore a lower cost may also be involved to build this turbine apart from producing more electrical power.

The dual-rotor counter or contra-rotation is a proven method in improving the performance of wind turbines substantially. Thus, in the present study, improving the conversion efficiencies, particularly in low-speed wind conditions are mainly sought throughout this study. This is in response to a persistent issue of most of the existing designs which fail to start moving or producing useful output at low wind speeds, or they are not meant for such conditions. Furthermore, given that the drag-type Savonius rotors are known for their low efficiency (relatively), they may be an optimal option when low wind speed is involved. This study, therefore, aims to explore for the first time the working conditions of a counter-rotating design based on drag-type Savonius VAWT while assessing its performance at a low upstream wind velocity. This counter-rotating technique is significant in VAWT designs, as it was able to enhance the power output of the H-type Darrieus rotor by almost threefold and corresponding conversion efficiency of $40 \%$ in previous literature [23], [24]. 
On the other hand, given that the current study seeks to operate the counter-rotating technique in low-speed wind conditions besides improving the conversion efficiency of the VAWT, the Savonius-type design with three blades is considered in this study for these aforementioned inherent characteristics which are in parallel with the objective of the present study beside taking advantage of the counter-rotating technique. Although there exist numerous studies investigating the performance of Savonius rotors, the present study could be considered the first to adopt the counterrotating concept on a Savonius-type rotor while providing a comprehensive assessment of this method and test the capability to operate at a lower wind speed range.

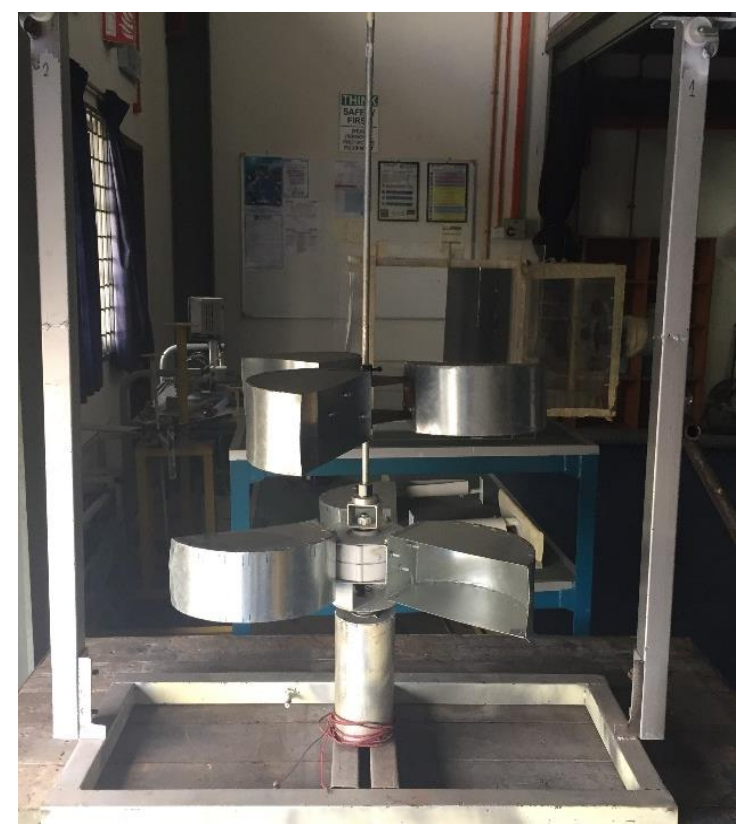

Fig. 1 - Actual photograph of the Savonius counter-rotating prototype

\section{Methodology}

\subsection{Savonius Counter-Rotating Prototype Design and Fabrication}

The two counter-rotating rotors of the present prototype are designed and fabricated based on the Savonius-type rotor concept. A 3D CAD model was initially developed and a complete model was later fabricated. The dimensional details for each rotor are shown in Table 1 and Figure 2. It should be noted that both the top and bottom rotors have similar dimensional sizes. Each rotor model is equipped with three blades, thus six blades in total. Each blade has dimensions of $12 \mathrm{~cm}, 10 \mathrm{~cm}$ and $27 \mathrm{~cm}$ for height, width and radius, respectively. The rotary motion of both rotors is counter-rotating with an orientation in a vertical axis. In order to achieve maximum efficiency of the rotors, the material of the rotor was carefully chosen by taking into account the weight and stiffness of the material as the material can affect the performance, safety and cost of the turbine. Thus, galvanized steel was chosen for all rotor blades for its excellent resistance to shock and ability to withstand high vibrations. However, for the shaft material, a $10 \mathrm{~cm}$ diameter aluminum rod was used. Moreover, the distance between the top rotor and the bottom rotor was fixed to $10 \mathrm{~cm}$ throughout the study.

Moreover, the upper rotor is connected to the shaft and is denoted as the primary rotor and the lower or bottom rotor is attached to the generator and is assigned as the secondary or auxiliary rotor, as illustrated in Figure 1 and Figure 4. It is worth noting that the rotation of the dual-rotor is fixed and each of them will spin in a predetermined direction so that a zero output would be avoided if they were to spin in the same direction. As such, the top (primary) rotor and the bottom (secondary) rotor spin, respectively in clockwise and counterclockwise directions. The fixing of counterrotating operation is achieved by using two unidirectional bearings; one installed at the tip of the shaft and another under the generator/alternator for the primary and secondary rotors, respectively to ensure rotation at clockwise and counterclockwise directions. Furthermore, given that the generator was moving alongside the bottom rotor, it was challenging to measure the electrical power output. As a result, a slip ring with a moving part on one side and a fixed part on the other side was installed below the generator. The moving parts of the slip ring are connected to the moving parts of the generator which are the wires in this case and the fixed parts are connected to the electric circuit board. Figure 2 below shows the geometrical details of the rotors, including views from different orientations as well as the main components of the current counter-rotating prototype. 
(a)

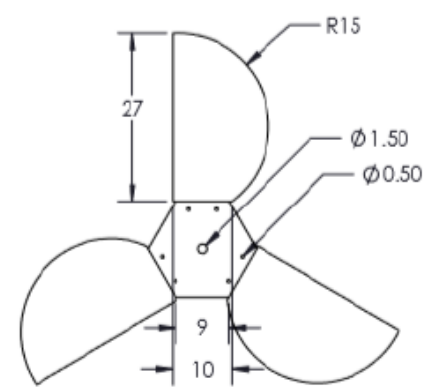

TOP VIEW

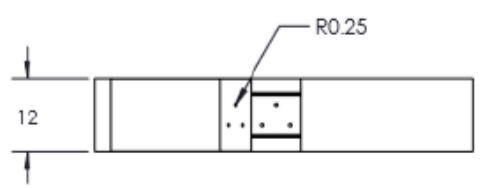

FRONT VIEW

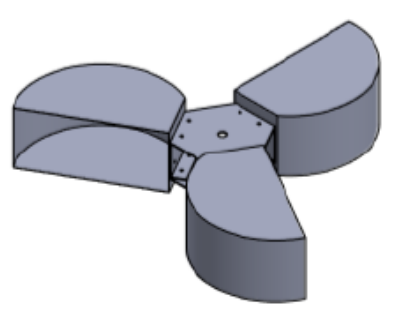

ISOMETRIC VIEW

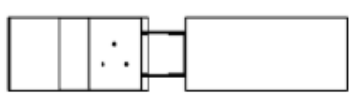

SIDE VIEW

(b)

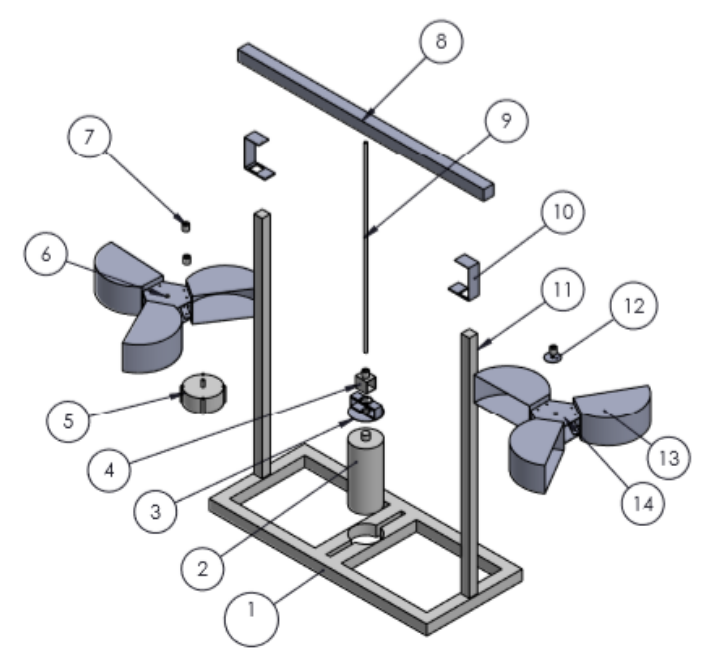

\begin{tabular}{|c|c|c|}
\hline Hem No. & Part Name & Quantity \\
\hline 1 & Base & 1 \\
\hline 2 & Tower & 1 \\
\hline 3 & Support & 1 \\
\hline 4 & $\begin{array}{c}\text { Generator } \\
\text { support }\end{array}$ & 1 \\
\hline 5 & Generator & 1 \\
\hline 6 & Lower rotor & 1 \\
\hline 7 & Holder & 4 \\
\hline 8 & Top pole & 1 \\
\hline 9 & Shaft & 1 \\
\hline 10 & Pole support & 2 \\
\hline 11 & Side pole & 2 \\
\hline 12 & Bearing & 2 \\
\hline 13 & Blade & 6 \\
\hline 14 & Upper rotor & 1 \\
\hline & & \\
\hline
\end{tabular}

Fig. 2 - Geometrical features of the model: (a) Dimensional details of a rotor and (b) Counter-rotating prototype componential details (Units are given in $\mathbf{c m}$ )

Table 1 - Model main specifications

\begin{tabular}{|c|c|c|c|}
\hline Features & Primary rotor & Secondary rotor & Unit \\
\hline Rotor type & Savonius-type & Savonius-type & \\
\hline Blade number & 3 & 3 & \\
\hline Rotor diameter, $\mathrm{D}$ & 0.54 & 0.54 & $\mathrm{~m}$ \\
\hline Rotor height, $\mathrm{H}$ & 0.12 & 0.12 & \\
\hline Rotation & Clockwise & Counterclockwise & \\
\hline Axial distance & 0.10 & & $\mathrm{~m}$ \\
\hline Wind speed condition, $\mathrm{v}$ & $2-9$ & $2-9$ & $\mathrm{~m} / \mathrm{s}$ \\
\hline
\end{tabular}

\subsection{Model Setup and Testing Procedure}

The performance investigations of the dual-rotor prototype were conducted at the wind tunnel facility available at Universiti Tun Hussein Onn Malaysia. The wind tunnel has a capacity of $10 \mathrm{hp}$ and 2880 rated RPM. The assessment is performed based on electrical power, torque, power coefficient and a torque coefficient, as shown in equations $1-4$. 
The testing of the newly fabricated dual-rotor configuration was conducted at the outlet of a wind tunnel. The testing location of the prototype has been tested several times before it was decided, in order to avoid any instability on the pressure and the velocity distributions around rotors. As shown in Figure 3, the distance of $0.55 \mathrm{~m}$ has shown to be the optimum location for the testing. The model was subjected to various wind velocities ranging from $2 \mathrm{~m} / \mathrm{s}$ to $9 \mathrm{~m} / \mathrm{s}$. The upstream velocity was quantified using a vane anemometer, while the angular rotational velocity was measured using a tachometer. Both have the accuracy of $\pm 1 \%$ and $\pm 0.03 \%$, respectively.

In order to rate the performance of the system and its low-speed wind operation capability, the electrical power output of the counter-rotating rotors was quantified by measuring the current and voltage of the rotors at each wind velocity, while using a digital multimeter with an accuracy of $\pm 0.5 \%$. It is to be noted that the capacity of the generator/alternator utilized is rated $100 \mathrm{~W}$ at $750 \mathrm{RPM}$ and its mass is $3.5 \mathrm{~kg}$. The complete setup of the system is demonstrated in detail in Figure 4.

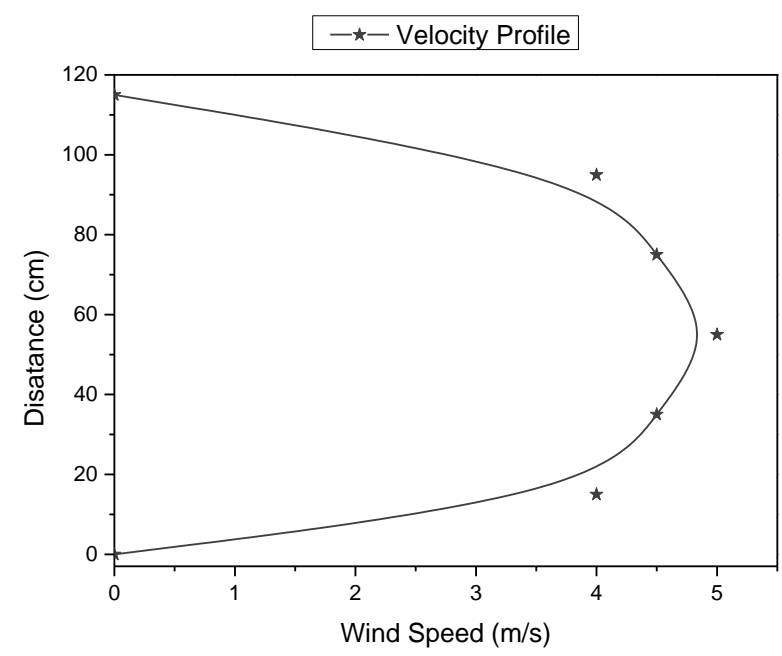

Fig. 3 - Velocity profile

$$
P=V \times I
$$

where $\mathrm{V}$ represents the voltage (volts) and I represents the current (ampere).

$$
\begin{aligned}
& T=\frac{30 P}{\Pi N} \\
& C_{P}=\frac{P}{0.5 \rho A V^{3}} \\
& C_{t}=\frac{2 T}{0.5 \rho A V^{2} r}
\end{aligned}
$$

where $\mathrm{N}$ and $\rho$ are respectively, the angular velocity of the rotor (RPM) in minutes and air density. Meanwhile, $r, A$ and $V$ represents the rotor radius, projected swept area $(\mathrm{H} \times \mathrm{D})$ and the upstream wind velocity, respectively. 


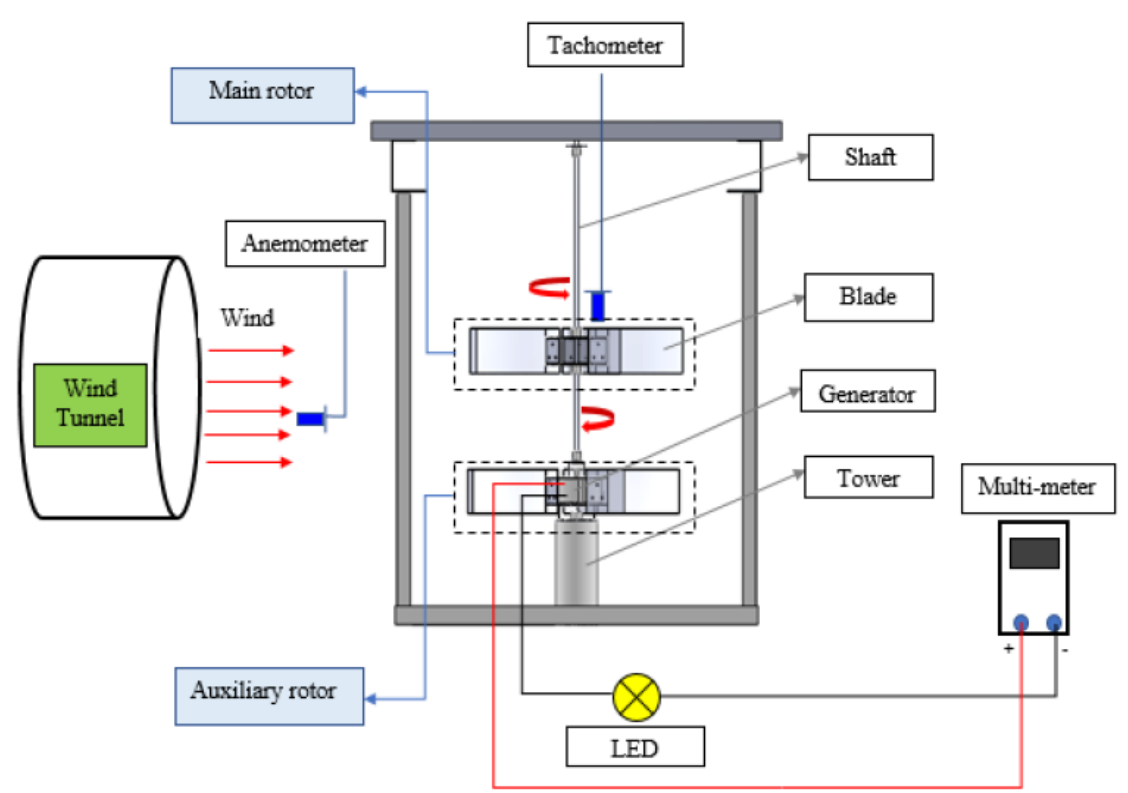

Fig. 4 - Counter-rotating model setup

\section{Experimental Results}

This segment discusses and brings together the electrical energy converted from the wind through to the counterrotation of the two rotors and the efficiency of such conversion.

\subsection{Rotational Speed (RPM)}

Measuring the rotational speed or the RPM of a wind turbine rotor is vital in the overall analysis of a wind turbine performance, especially when a small generator is involved in extracting the kinetic energy from the wind into usable electricity. This is simply because the electrical output of a wind turbine generator increases proportionally with rotational speed. As such, the performance of the present turbine is estimated in terms of the rotational speed for the two counter-rotating rotors with respect to a working flow condition in the range between $2 \mathrm{~m} / \mathrm{s}$ and $9 \mathrm{~m} / \mathrm{s}$. This is particularly useful given that the secondary rotor (bottom rotor) is articulated to the generator.

By referring to the graph shown in Figure 5, it is clear that both rotors started spinning from as low as $2 \mathrm{~m} / \mathrm{s}$ and generated useful electrical power at this cut-in speed. However, the rotational speed that emerged from each rotor varies notably. The graph also highlights the consistent increase in the rotational speed of both rotors with increased wind speed. The CRWT system has the highest values compared to the individual rotors. This is due to the summation of primary and secondary rotors of the CRWT system values. In fact, the RPM has risen by up to $90 \%$ due to the presence of twin rotors, which clearly signifies increased power output. This clearly proves the significance of the additional auxiliary rotor in the system. Moreover, higher RPM also indicates more power output as long as the rotational speed is within the rated RPM of the generator being used since the power produced from the kinetic energy of wind via a wind turbine rotor through torque shows a direct correlation with torque and RPM of the rotor as in Equation 2. However, in the cases where the rotational velocity exceeds the rated RPM of the generator, the output remains constant even though the rotational speed is being increased due to this limit.

Furthermore, it can also be observed that the rotational speed at $9 \mathrm{~m} / \mathrm{s}$ holds the highest values for both rotors. Maximum RPM of up to 205 and 179, were respectively achieved from the primary and secondary rotors and therefore, the output of the CRWT in terms of RPM was as high as 384 RPM. However, the lowest RPM was witnessed at $2 \mathrm{~m} / \mathrm{s}$ upstream wind velocity, which was considered as the start-up speed. In this condition, both rotors exhibited the lowest rotational speeds where the primary rotor and secondary rotor were 57 RPM and 42 RPM, respectively.

It is also interesting to note that although the primary and secondary rotors were subjected to a similar upstream velocity, the angular velocity stemmed from the two rotors was different. The RPM of the primary rotor was higher compared to the secondary rotor RPM. Consequently, the electrical power output from the main rotor was higher. This could mainly be owed to inertia encountered by the auxiliary rotor due to the $5 \mathrm{~kg}$ weight of the generator. This behaviour is also in agreement with the previous study carried out in [11]. 


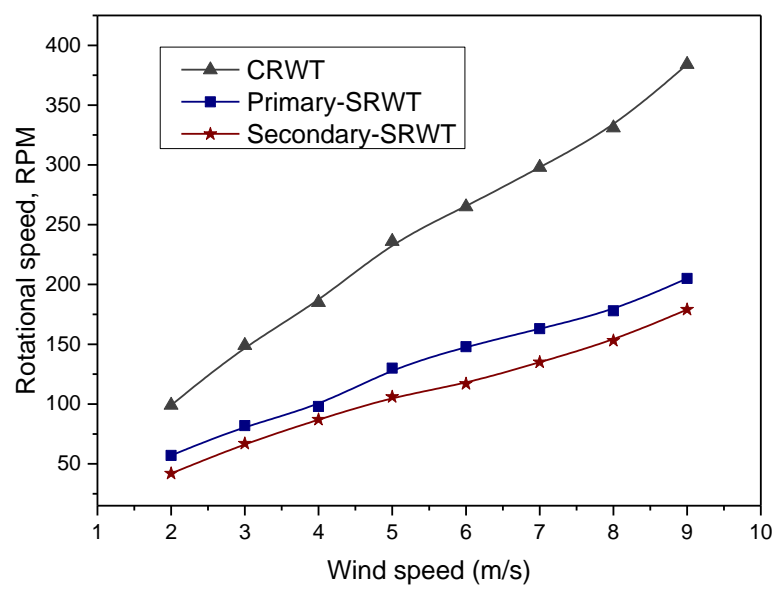

Fig. 5 - Comparison of RPM against upstream wind velocity

\subsection{Torque Output}

The performance of the current prototype is evaluated in terms of the main wind turbine rotor characteristics including torque, torque coefficient and power coefficient. The torque output is predicted from the electrical power obtained from the counter-rotating rotors. As shown in Figure 6, the torque output from the two rotors, including the Betz limit is presented with respect to the wind speed range covered. It is highlighted that constant growth in torque output is observed as the free-stream velocity rises. Comparing the amount produced by two rotors, the primary rotor generated more torque than the secondary rotor over the entire wind speed conditions covered. This difference becomes more evident in higher wind speed conditions than in lower ones. The peak outputs from the top and bottom rotors were observed at $9 \mathrm{~m} / \mathrm{s}$ with corresponding torque outputs of $0.36 \mathrm{Nm}$ and $0.27 \mathrm{Nm}$, respectively.

The improved performance emerged from the primary rotor is owed to higher RPM, which resulted from the smooth operation, unlike the secondary rotor, which has to produce more thrust to spin the generator. However, the net output of the torque generated by these rotors is significant due to the presence of the auxiliary rotor. Thus, the total maximum torque output achieved was $0.63 \mathrm{Nm}$ at $9 \mathrm{~m} / \mathrm{s}$ wind speed.

Moreover, based on Figure 6, the total output values of the CRWT are in agreement with the Betz limit, where they were observed to be not far from theoretical values, notably in lower wind conditions as illustrated below in Figure 6. It is interesting to note that while a single-rotor system could have achieved just an equitable output, the existence of the second rotor has improved the output by almost $42 \%$ extra torque on average than a single-rotor system as shown in Figure 7. The corresponding average torque coefficient was $42 \%$ and the power coefficient was $28 \%$. This clearly indicates the effectiveness of this method over a single-rotor system in improving the performance of the system.

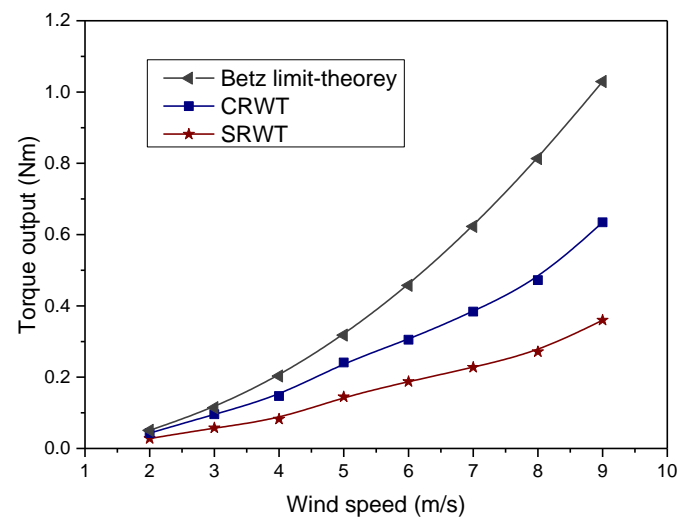

Fig. 6 - Comparison of torque outputs of the model with Betz limit

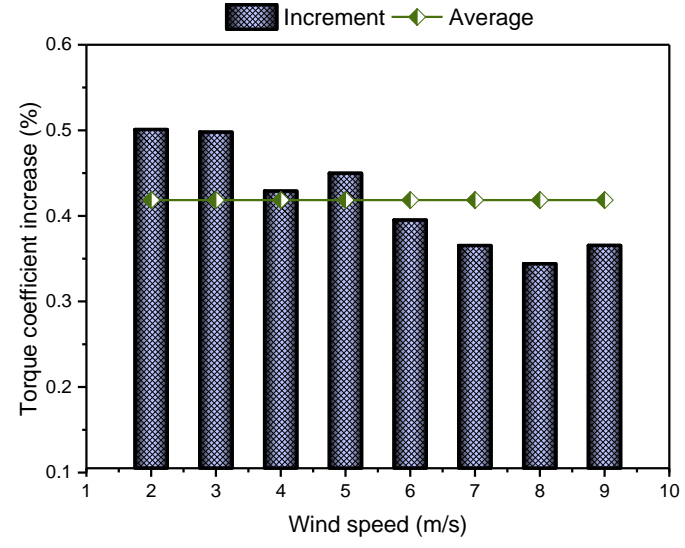

Fig. 7 - Percentage increase of torque for the new system at each wind speed

\subsection{Power Coefficient}

Typically, a Savonius wind turbine has an inherent capability to perform better under a low-velocity condition than a higher wind condition. In this experiment, aerodynamic coefficients such as power coefficient, Cp and torque 
coefficient, $\mathrm{Ct}$ are predicted. As the estimation of these quantities is necessary while establishing an extensive assessment of the CRWT system in order to characterize its performance in the required wind condition. Thus, the conversion efficiency of the model in terms of power coefficient is presented in this part over the entire wind speed range covered $(2 \mathrm{~m} / \mathrm{s}-9 \mathrm{~m} / \mathrm{s})$.

As shown in Figure 8, it is indicated that the power coefficient, $\mathrm{Cp}$ varies notably with the wind speed. Overall, the conversion efficiency of the dual-rotor turbine is high at the low-speed wind and tends to decline as the wind velocity rises. The same behaviour was observed in the primary rotor as well as the secondary rotor, as demonstrated in Figure 8. This phenomenon happens owing to the inherent difficulties of the Savonius rotor to convert effectively at increased operating speeds. Therefore, it is usually found in applications that require lower wind speed and higher torque. The maximum efficiencies reached up to $27 \%$ and $12 \%$, respectively for the primary and secondary rotors. Consequently, the maximum efficiency of the current counter-rotating turbine has peaked at $38 \%$ due to this technique.

However, although the individual output from the two counter-rotating rotors could be considered low, the combination of the two rotors results in a substantial increment in the output, which reflects on the significance of this method in enhancing the conversion efficiency or the performance of the system. Thus, the average percentage increase of the power coefficient over the entire wind conditions covered reaches approximately $28 \%$. However, comparing this average increase with the previous efficiencies achieved using the same counter-rotating concept, but in an H-type CRWT [11], the current efficiency is lesser. This is understandable as an H-type rotor is inherently more efficient than a Savonius-type rotor but showed difficulties to start spinning in low-speed wind conditions. Nevertheless, the additional amount obtained due to this concept in a Savonius-type is impressive and opens many potentials for this technique to have a genuine impact on wind energy harvesting fields. Furthermore, the present configuration has also the advantage to operate at a much lower upstream wind velocity compared to the previous H-type design. This is a significant attribute and more convenient for sites with low and average wind speed conditions.

\subsection{Torque Coefficient}

Torque coefficient, $\mathrm{Ct}$ is one of the essential aspects while evaluating the performance of a wind turbine. Thus, the conversion efficiency of the current system is estimated based on torque coefficient with regard to a low working flow condition, which starts from $2 \mathrm{~m} / \mathrm{s}$. As illustrated in Figure 9, a constant decrease in the values of Ct is observed as the working velocity increases. This is true for both the primary rotor and the secondary rotor. This denotes that the current design could perform better in lower wind conditions than higher operating conditions. In other words, the system may not encounter self-starting difficulties besides better conversion efficiency in this condition. However, it can be generally observed that both the primary rotor and secondary rotor produced high torque at the low-speed wind and lower at high wind speeds, which led to higher conversion efficiencies in this condition. Nevertheless, the increment of the efficiencies for both rotors was small.

Furthermore, as revealed in Figure 9, the current design predicts approximately $20-32 \%$ efficiency with respect to the primary rotor and about $15-20 \%$ with regard to the secondary rotor. This low efficiency could be attributed to mechanical wear losses, losses due to the wake of the rotor, blade tip losses, drag due to boundary layer and non-ideal flow. However, by looking at the net output from two contrary rotating rotors, a considerable performance enhancement was possible owing to the addition of the second rotor in the system. The minimum and maximum efficiency of the new design reach up to $34 \%$ and $50 \%$, respectively, which is not possible for a conventional Savonius SRWT. The average percentage increment of approximately $42 \%$ in the conversion efficiency was attainable due to this technique.

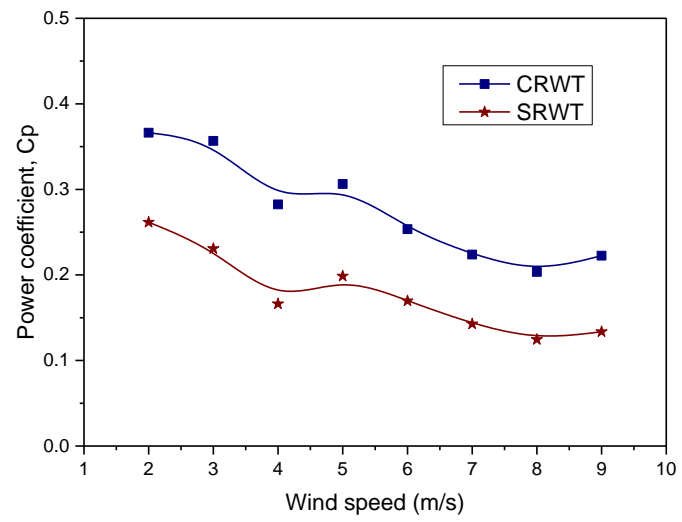

Fig. 8 - Power coefficients against upstream wind velocity

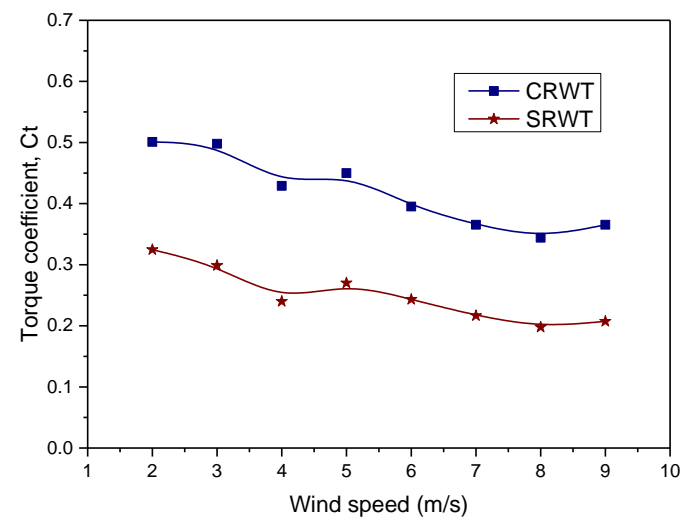

Fig. 9 - Torque coefficient against upstream wind velocity 


\section{Conclusion}

In this paper, an experimental study of a small Savonius-type VAWT based on the counter-rotating concept was carried. The purpose of this work was to investigate the capability of this innovative technique in improving the performance of the Savonius-rotor wind turbine and the effectiveness to operate at a poor upstream wind velocity. The performance examination of the system was established based on aerodynamic quantities, including power coefficient and torque coefficient for a working flow condition that ranges between $2 \mathrm{~m} / \mathrm{s}$ and $9 \mathrm{~m} / \mathrm{s}$.

It is indicated that the new counter-rotating VAWT has enhanced the performance of the system expressively and was able to operate effectively at a lower upstream wind velocity starting from a cut-in velocity of $2 \mathrm{~m} / \mathrm{s}$. Moreover, the primary top rotor performed better compared to the bottom secondary rotor. However, the presence of the second rotor was quite effective and thus a performance improvement of almost $42 \%$ was achieved on average in terms of torque output as compared to a configuration with a single rotor. Furthermore, the results also showed that the conversion efficiency of the system had been enhanced substantially. The average power coefficient achieved was $28 \%$, meanwhile, the torque coefficient has reached $42 \%$ due to the existence of the auxiliary rotor in the new configuration. This technique is therefore thought to be quite promising for wind energy conversion devices particularly for sites with poor wind conditions in general. The potential sites for small-scale applications of the current model may include turbulent urban environment such as the rooftops of buildings, LED lighting for roads, etc. Meanwhile, rural areas with no grid connection, highways and offshore projects could be convenient for large-scale applications.

\section{Acknowledgment}

This research was funded by Universiti Tun Hussein Onn Malaysia through industrial grant with the grant number M002.

\section{References}

[1] B. Abdulameer et al., "Review on Diffuser Augmented Wind Turbine (DAWT)," International Journal of Integrated Engineering, vol. 11, no. 1, pp. 178-206, 2019, doi: 10.30880/ijie.2019.11.01.021

[2] A. Bianchini et al., "An Experimental and Numerical Assessment of Airfoil Polars for Use in Darrieus Wind Turbines-Part II: Post-stall Data Extrapolation Methods," Journal of Engineering for Gas Turbines and Power, vol. 138, no. 3, p. 032603, 2015, doi: 10.1115/1.4031269

[3] M. H. Mohamed, "Performance investigation of H-rotor Darrieus turbine with new airfoil shapes," Energy, vol. 47, no. 1, pp. 522-530, 2012, doi: 10.1016/j.energy.2012.08.044

[4] A. R. El-Baz, K. Youssef, and M. H. Mohamed, "Innovative improvement of a drag wind turbine performance," Renewable Energy, vol. 86, pp. 89-98, Feb. 2016, doi: 10.1016/j.renene.2015.07.102

[5] E. Pap, M. H. Mohamed, D. Thévenin, and G. Janiga, "Optimization of Savonius turbines using an obstacle shielding the returning blade," Renewable Energy, vol. 35, no. 11, pp. 2618-2626, 2010, doi: 10.1016/j.renene.2010.04.007

[6] M. H. Mohamed, G. Janiga, E. Pap, and D. Thévenin, "Optimal blade shape of a modified Savonius turbine using an obstacle shielding the returning blade," Energy Conversion and Management, vol. 52, no. 1, pp. 236242, 2011, doi: 10.1016/j.enconman.2010.06.070

[7] D. Gertz and D. A. Johnson, "An evaluation testbed for wind turbine blade tip designs-baseline case," International Journal of Energy Research, vol. 35, no. 15, pp. 1360-1370, 2011

[8] D. H. Didane, S. Mohd, Z. Subari, N. Rosly, M. F. Zulkifli, and M. F. Masrom, “An aerodynamic performance analysis of a perforated wind turbine blade," IOP Conference Series: Materials Science and Engineering, vol. 160, p. 012039, 2016, doi: 10.1088/1757-899X/160/1/012039

[9] H. Silaghi and V. Spoială, "PM wind generator topologies," Journal of Electrical and Electronics Engineering, vol. 1, no. 1, pp. 125-129, 2008, doi: 10.1109/TIA.2005.858261

[10] J. D. Booker, P. H. Mellor, R. Wrobel, and D. Drury, "A compact, high efficiency contra-rotating generator suitable for wind turbines in the urban environment," Renewable Energy, vol. 35, no. 9, pp. 2027-2033, 2010, doi: 10.1016/j.renene.2010.02.003

[11] D. H. Didane, N. Rosly, M. F. Zulkafli, and S. S. Shamsudin, "Performance Evaluation of a Novel Vertical Axis Wind Turbine with Coaxial Contra-Rotating Concept," Renewable Energy, vol. 115, pp. 353-361, 2018, doi: 10.1016/j.renene.2017.08.070

[12] N. J. Lee, I. C. Kim, C. G. Kim, B. S. Hyun, and Y. H. Lee, "Performance study on a counter-rotating tidal current turbine by CFD and model experimentation," Renewable Energy, vol. 79, pp. 122-126, 2015, doi: 10.1016/j.renene.2014.11.022

[13] S. Lee, E. Son, and S. Lee, "Velocity interference in the rear rotor of a counter-rotating wind turbine," Renewable Energy, vol. 54, pp. 235-240, 2013, doi: 10.1016/j.renene.2012.08.003

[14] S. N. Jung, T. S. No, and K. W. Ryu, "Aerodynamic performance prediction of a $30 \mathrm{~kW}$ counter-rotating wind turbine system,” Renewable Energy, vol. 30, no. 5, pp. 631-644, 2005, doi: 10.1016/j.renene.2004.07.005 
[15] T. Chaichana and S. Chaitep, "Performance Evaluation of Co-Axis Counter-Rotation Wind Turbine," Energy Procedia, vol. 79, pp. 149-156, 2015, doi: 10.1016/j.egypro.2015.11.453

[16] S. Merchant, J. Gregg, K. Van Treuren, and I. Gravagne, "Wind Tunnel Analysis of a Counter-Rotating Wind Turbine," 2009, [Online]. Available: http://web.ecs.baylor.edu/faculty/gravagnei/archived/ASEE_2009-TB3 1.pdf

[17] R. W. Y. Habash, V. Groza, Y. Yang, C. Blouin, and P. Guillemette, "Performance of a Contrarotating Small Wind Energy Converter," ISRN Mechanical Engineering, vol. 2011, pp. 1-10, 2011, doi: 10.5402/2011/828739

[18] A. I. Hassane, D. H. Didane, A. M. Tahir, and J. Hauglustaine, "Wind and Solar Assessment in the Sahelian Zone of Chad," International Journal of Integrated Engineering, vol. 10, no. 8, pp. 203-213, 2018

[19] D. H. Didane, A. A. Wahab, S. S. Shamsudin, N. Rosly, M. F. Zulkafli, and S. Mohd, "Assessment of wind energy potential in the capital city of Chad, N'Djamena," in AIP Conference Proceedings, 2017, vol. 1831, p. 020049, doi: 10.1063/1.4981190

[20] S. Mathew, Wind energy: Fundamentals, resource analysis and economics. 2006

[21] B. G. Newman, "Actuator-disc theory for vertical-axis wind turbines," Journal of Wind Engineering and Industrial Aerodynamics, vol. 15, no. 1-3, pp. 347-355, 1983, doi: 10.1016/0167-6105(83)90204-0

[22] H. J. Sutherland, D. E. Berg, and T. D. Ashwill, "A Retrospective of VAWT Technology," Security, no. January, pp. 1-64, 2012, doi: 10.2172/1035336

[23] D. H. Didane, S. M. Maksud, M. F. Zulkafli, N. Rosly, S. S. Shamsudin, and A. Khalid, "Performance investigation of a small Savonius-Darrius counter-rotating vertical-axis wind turbine," International Journal of Energy Research, vol. 44, no. 12, pp. 9309-9316, 2020, doi: 10.1002/er.4874

[24] D. H. Didane, S. M. Maksud, M. F. Zulkafli, N. Rosly, S. S. Shamsudin, and A. Khalid, "Experimental Study on the Performance of a Savonius-Darrius Counter-Rotating Vertical Axis Wind Turbine," IOP Conference Series: Earth and Environmental Science, vol. 268, p. 012060, 2019, doi: 10.1088/1755-1315/268/1/012060 